\title{
Thermal conductivity of single and multi-stacked DI-BSCCO tapes
}

\author{
Tomoyuki Naito $^{a}$, Hiroyuki Fujishiro ${ }^{a}$, Yuichi Yamada ${ }^{b}$ \\ ${ }^{a}$ Faculty of Engineering, Iwate University, Morioka 020-8551, Japan \\ ${ }^{b}$ Sumitomo Electric Industries, Ltd., 1-1-3 Shimaya, Konohana-ku, Osaka 554-0024, \\ Japan
}

\section{Abstract}

We have measured the temperature dependence of the thermal conductivity, $\kappa(T)$, for DI-BSCCO ${ }^{\circledR}$ tapes fabricated by Sumitomo Electric Industries, Ltd., which are $(\mathrm{Bi}, \mathrm{Pb})_{2} \mathrm{Sr}_{2} \mathrm{Ca}_{2} \mathrm{Cu}_{3} \mathrm{O}_{8+x}$ tapes sheathed with $\mathrm{Ag}$ or $\mathrm{Ag}-\mathrm{Au}$ alloy. The $\kappa(T)$ of the tape sheathed with $\mathrm{Ag}$ (residual resistance ratio $(\mathrm{RRR})=15)$ decreases with decreasing temperature and starts to increase rapidly below $60 \mathrm{~K}$, with a maximum at around $15 \mathrm{~K}$. On the other hand, the $\kappa(T)$ of the tape sheathed with $\mathrm{Ag}-5.4 \mathrm{wt} \% \mathrm{Au}$ alloy has a very low value that decreases monotonically with decreasing temperature. At around $77 \mathrm{~K}$, the absolute values of $\kappa(T)$ for both tapes were about $260 \mathrm{~W} \mathrm{~m}^{-1} \mathrm{~K}^{-1}$ and $80 \mathrm{~W} \mathrm{~m}^{-1} \mathrm{~K}^{-1}$, respectively. The $\kappa(T)$ of a stacked sample, in which six DI-BSCCO tapes sheathed with Ag are soldered, was also measured. The measured $\kappa(T)$ was fairly well reproduced by the estimated $\kappa(T)$, which was calculated using the measured $\kappa(T)$ of the single tape and solder.

Key words: High Tc superconductors (A), Thermal conductivity (C), Transport properties (C) 


\section{Introduction}

A superconducting tape consisting of $(\mathrm{Bi}, \mathrm{Pb})_{2} \mathrm{Sr}_{2} \mathrm{Ca}_{2} \mathrm{Cu}_{3} \mathrm{O}_{8+x}$ (Bi2223) superconductor and an Ag sheath, called Bi2223/Ag, has been studied by many researchers and companies. Sumitomo Electric Industries, Ltd. has developed a new sintering technique with a controlled overpressure (CTOP) [1], which enables us to fabricate the Bi2223/Ag tape without voids and with an extremely small amount of non-superconducting phases. Recently, using this method, a Bi2223/Ag tape with a high critical current over $200 \mathrm{~A}$ at $77 \mathrm{~K}$ and under the self-field was successfully manufactured [2]. Moreover, it has a higher modulus of elasticity than conventional Bi2223/Ag tape [3]. Therefore, it was named Dynamically Innovative BSCCO (DI-BSCCO $\left.{ }^{\circledR}\right)$.

The critical current over 200 A allows for the possibility of using the tape practically in superconducting devices such as high-field coil magnets and power cables in liquid $\mathrm{N}_{2}$. In this case, however, we must correctly estimate the amount of heat leakage through the tape [4]. The heat leakage

$Q^{\text {leak }}$ through the tape between the low and high temperature sides, $T^{\text {low }}$ and $T^{\text {high }}$, respectively, is generally given by

$$
Q^{\text {leak }}=\frac{S}{L} \int_{T^{\text {low }}}^{T^{\text {high }}} \kappa(T) d T,
$$

where $\kappa(T)$ is the temperature dependence of the thermal conductivity, $L$ the length and $S$ the cross section of the tape. The absolute value and the temperature dependence of $\kappa(T)$ strongly depend on the sheath material, the sheath/Bi2223 ratio and the fabrication process $[4,5,6,7]$. As far as we know, the $\kappa(T)$ of DI-BSCCO has not been reported.

In this paper, we report the temperature dependence of the thermal con- 
ductivity, $\kappa(T)$, for two types of DI-BSCCO tapes. The thermal conductivity was also measured for a stacked sample consisting of six tapes that were joined by a commercial solder, and the $\kappa(T)$ obtained for the stacked sample was compared with the $\kappa(T)$ calculated using the measured thermal conductivities of both the single tape and the solder.

\section{Experimental}

DI-BSCCO tapes were fabricated by the powder in tube (PIT) method with the CT-OP sintering technique. The details of sample preparation have been described elsewhere [1]. Two types of DI-BSCCO tapes (types H and G) were studied. A stacked sample was also prepared, consisting of six type $\mathrm{H}$ tapes that were joined by the commercial solder (LFM-48). Hereafter, we refer to this sample as type H6. The sheath material of type $\mathrm{H}$ is Ag. We estimate the purity of $\mathrm{Ag}$ using a residual resistance ratio (RRR), because the thermal conductivity of the metal strongly depends on the purity. Figure 1 shows the temperature dependence of the reduced resistance $R / R(11.5 \mathrm{~K})$ for $\mathrm{Ag}$ of type $\mathrm{H}$ tape. The value of $\mathrm{RRR}$ was estimated to be about $R(300$ $\mathrm{K}) / R(11.5 \mathrm{~K})=15$. The inset of Fig. 1 shows the RRR vs. impurity content for high purity copper, which is reported in Ref. [8]. Assuming that a similar relation is realized for silver, the purity of $\mathrm{Ag}$ used for type $\mathrm{H}$ tape is roughly estimated to be $99.9 \%$ by extrapolating the curve, which is indicated by the arrow in the figure. The sheath material of type $\mathrm{G}$ tape is an Ag-5.4wt\% Au alloy. Figure 2 is a picture of the cross section of the type $\mathrm{H}$ tape. From this picture, the sheath/Bi2223 ratio of type $\mathrm{H}$ tape was estimated to be about 1.8. The sheath/Bi2223 ratio of the tape of type $G$ was almost the same 
value. Table 1 summarises the specifications of the DI-BSCCO tapes.

The thermal conductivity along the surface of the tape, i.e., along the $a b$ plane of the Bi2223 filament, was measured by a conventional steady-state heat flow method [9]. The experimental setup is schematically illustrated in the inset of Fig. 3. One end of the tape was soldered to the copper stage, and a small metal chip resistor $(1 \mathrm{k} \Omega)$ was adhered to the other end of the tape as a heater using GE7031 varnish. The $\kappa$ is estimated by the relation

$$
\kappa=\frac{Q}{\Delta T} \cdot \frac{L}{S}
$$

where $Q$ is the applied heat flow, $\Delta T$ is the temperature difference, $L$ is the distance between the thermocouples, and $S$ is the cross section of tape. $\Delta T$ was measured using two Chromel-Constantan thermocouples $(76 \mu \mathrm{m}$ in diameter) that were adhered to the sample with GE7031 varnish. The upper thermocouple was set at a distance of about $5 \mathrm{~mm}$ from the heater, and $L$ was typically about $10 \mathrm{~mm}$. The length of each tape was about $25 \mathrm{~mm}$. $Q$ was generated by flowing current to the chip resistor and was automatically controlled to maintain a $\Delta T$ of $0.5-0.8 \mathrm{~K}$. The electrical resistivity was measured by a four-probe method with a typical current density of about $3 \mathrm{~A} / \mathrm{cm}^{2}$. The temperature of copper stage was controlled using a GiffordMcMahon cycle helium refrigerator from 5 to $300 \mathrm{~K}$. To eliminate radiation loss, the thermal conductivity was measured below $250 \mathrm{~K}$.

\section{Results and Discussion}

\subsection{Thermal conductivity for single tapes}

Figure 3 shows the temperature dependence of the thermal conductivity, $\kappa(T)$, for types $\mathrm{H}$ and G DI-BSCCO tapes. The $\kappa(T)$ of type $\mathrm{H}$ tape decreases 
slightly with decreasing temperature from $250 \mathrm{~K}$ to $100 \mathrm{~K}$. It increases moderately between $100 \mathrm{~K}$ and $60 \mathrm{~K}$, starts to increase rapidly below $60 \mathrm{~K}$, and has a peak around $15 \mathrm{~K}$. The peak value is approximately $800 \mathrm{~W} \mathrm{~m}^{-1} \mathrm{~K}^{-1}$. On the other hand, the $\kappa(T)$ of the type $\mathrm{G}$ tape decreases monotonically with decreasing temperature. The absolute value of $\kappa$ for the type $\mathrm{G}$ tape near $77 \mathrm{~K}$ is about one third of that for type $\mathrm{H}$ tape. No anomaly appears at the superconducting transition in either sample. The shape of the $\kappa(T)$ curve for type $\mathrm{H}$ tape is similar to that for a high-purity metallic conductor. The thermal conductivity for a part of the sheath, $\kappa_{\text {sheath }}$, can be estimated from the relation

$$
\kappa_{\text {tape }}=f_{\text {sc }} \kappa_{2223}+\left(1-f_{\text {sc }}\right) \kappa_{\text {sheath }}
$$

where $\kappa_{\text {tape }}$ and $\kappa_{2223}$ are the thermal conductivities for the whole tape and the Bi2223 filaments, respectively, and $f_{\mathrm{sc}}$ is the volume ratio of the Bi2223 parts to the whole tape, the value of which is roughly estimated to be 0.36 for type $\mathrm{H}$ tape using the sheath/Bi2223 ratio (=1.8). Using this relation, the peak value of $\kappa_{\text {sheath }}$ for type $\mathrm{H}$ tape is approximately estimated to be $1200 \mathrm{~W} \mathrm{~m}^{-1} \mathrm{~K}^{-1}$. Here, we use the reported values of $\kappa_{2223}$ for polycrystalline Bi2223 [9]. This value approximately corresponds to that for Ag-0.09at\% Au alloy [10], indicating that the purity of $\mathrm{Ag}$ for type $\mathrm{H}$ tape is better than $99.9 \%$. This value is consistent with the purity estimated from the value of RRR in Sec. 2. Consequently, we can conclude that the sharp peak in $\kappa(T)$ for type $\mathrm{H}$ tape originates from the nature of the high-purity metal. On the other hand, the small value of $\kappa(T)$ for type $\mathrm{G}$ tape is often observed in a solid solution. The behaviours of $\kappa(T)$ observed here resemble those of other Bi2223 tapes sheathed with silver-based alloys $[4,5,6,7]$. 
In a conducting solid, the thermal conductivity generally consists of contributions from both phonons and electrons, $\kappa_{\mathrm{ph}}$ and $\kappa_{\mathrm{el}}$. Using the electrical resistivity, $\kappa_{\mathrm{el}}$ is roughly estimated by the Wiedemann-Franz (W-F) law, $\kappa_{\mathrm{el}}=L_{0} T / \rho$, where $L_{0}\left(=2.45 \times 10^{-8} \mathrm{~W} \Omega^{-1} \mathrm{~K}^{-1}\right)$ is the Lorenz number. Figure 4 shows the temperature dependence of the electrical resistivity, $\rho(T)$, for types $\mathrm{H}$ and $\mathrm{G}$ DI-BSCCO tapes. The critical temperature, $T_{\mathrm{c}}$, as determined by zero electrical resistivity, was about $111 \mathrm{~K}$ for both samples, demonstrating the high quality of the superconducting part of Bi2223. Both $\rho(T)$ curves show a good linear dependence on $T$ above $T_{\mathrm{c}}$. The absolute value of $\rho(T)$ for type $\mathrm{G}$ tape is somewhat larger than that of type $\mathrm{H}$, because the sheath metal of the former is a solid solution Ag-Au alloy (Ag-5.4wt\% Au alloy). Considering the linear dependence on $T$ of the $\rho(T)$ and the W-F law, the $\kappa_{\mathrm{el}}$ is expected to be independent of temperature. As a result, the values of $\kappa_{\mathrm{el}}$ for each sample are estimated to be about 240 (type H) and $110 \mathrm{~W} \mathrm{~m}^{-1} \mathrm{~K}^{-1}$ (type $\mathrm{G}$ ), which are shown as the broken lines in Fig. 3. These values are comparable to the measured $\kappa(T)$ values, indicating that the dominant heat carriers are electrons. In the normal state, the applied current mainly flows through the metal sheath, because the resistivities of the metal sheaths are considerably smaller than that of Bi2223; for instance, the reported value of $\rho$ at $150 \mathrm{~K}$ for Bi2223 polycrystal is about $500 \mu \Omega \mathrm{cm}[9]$ and that for pure silver is $0.5 \mu \Omega \mathrm{cm}$ [5]. On the other hand, the $\kappa$ values of polycrystalline Bi2223 below $150 \mathrm{~K}$ were reported to be smaller than approximately $3 \mathrm{~W} \mathrm{~m}^{-1} \mathrm{~K}^{-1}[9]$. The $\rho(T)$ of polycrystalline Bi2223 also shows the metallic linear dependence on $T$, and therefore $\kappa_{\text {el }}$ was estimated to be about $0.8 \mathrm{~W} \mathrm{~m}^{-1} \mathrm{~K}^{-1}[9]$ in the normal state, which is approximately 20 
$\%$ of the total $\kappa$, meaning that the dominant heat carriers are phonons in Bi2223. Consequently, in both the superconducting and normal states, the contribution of the Bi2223 filaments to the heat transport is negligibly small, and most of the applied heat is carried by electrons through the metal sheath. We previously reported that the behaviour of the $\kappa(T)$ of the Bi2223/Ag-Au tape is quite similar to that of the $\mathrm{Ag}-\mathrm{Au}$ alloy [5], which is consistent with the present results.

\subsection{Thermal conductivity of a stacked sample}

Figure 5(a) shows the temperature dependence of the thermal conductivities, $\kappa_{\text {mes }}^{(1)}(T), \kappa_{\text {mes }}^{(6)}(T)$ and $\kappa_{\text {mes }}^{\text {sol }}(T)$, for the single tape (type $\mathrm{H}$ ), sixfold sample (type H6) and solder (LFM-48), respectively. $\kappa_{\text {mes }}^{(6)}(T)$ almost coincides with $\kappa_{\text {mes }}^{(1)}(T)$. The absolute value of $\kappa_{\text {mes }}^{\text {sol }}(T)$ is somewhat smaller than both $\kappa_{\text {mes }}^{(1)}(T)$ and $\kappa_{\text {mes }}^{(6)}(T)$, but increases very slowly with decreasing temperature from $250 \mathrm{~K}$ to around $40 \mathrm{~K}$. Below $40 \mathrm{~K}$, it increases somewhat rapidly with decreasing temperature.

We attempted to estimate the $\kappa(T)$ value for $n$-fold sample using those of the single tape and the solder. The total thermal conductivity, $\kappa^{(n)}$, is written with the relation

$$
\kappa^{(n)}=\frac{Q^{(n)}}{\Delta T} \cdot \frac{L}{S^{(n)}},
$$

where $Q^{(n)}$ is total applied heat flow and $S^{(n)}$ is the cross section of the stacked sample. The schematic image of heat flow through the stacked sample is demonstrated in the inset of Fig. 5(a). The stacked $n$-fold sample consists of $n$ pieces of tape and $(n-1)$ layers of solder. Here, we denote the heat flow and the thermal conductivity for the single tape as $Q^{(1)}$ and $\kappa^{(1)}$ and 
those for the solder as $Q^{\text {sol }}$ and $\kappa^{\text {sol }}$, respectively. In this configuration, the equivalent circuit is parallel to that of the heat transport. Thus, the $Q^{(n)}$ is given as

$$
Q^{(n)}=n Q^{(1)}+(n-1) Q^{\mathrm{sol}}
$$

Since the $\Delta T$ and $L$ are the same for all parts, $\kappa^{(n)}$ is written as

$$
\kappa^{(n)}=\left(\kappa^{(1)} \times n S^{(1)}+\kappa^{\mathrm{sol}} \times S^{\mathrm{sol}}\right) / S^{(n)},
$$

where $S^{\text {sol }}$ is the sum of cross section for the solder part, i.e., $S^{\text {sol }}=S^{(n)}-$ $n S^{(1)}$. In this case, $S^{\text {sol }}$ was estimated to be $0.27 \mathrm{~mm}^{2}\left(=S^{(6)}-6 \times S^{(1)}\right)$, which is approximately $5 \%$ of the total cross section of type H6 tape. We also calculated the thermal conductivity of type $\mathrm{H} 6$ tape, which is denoted as $\kappa_{\text {cal }}^{(6)}$ and depicted in Fig. $5(\mathrm{a}) . \kappa_{\text {cal }}^{(6)}(T)$ nearly corresponds to the measured $\kappa_{\text {mes }}^{(6)}(T)$, meaning that the thermal conductivity of the $n$-fold sample can be estimated using those of the single tape and the solder.

Using this relation, the $\kappa(T)$ 's of type G (single tape) and the solder give the $\kappa_{\text {cal }}^{(6)}$ of a six-fold sample of type $G$ tape, which does not exist at present. Here, the value of $S^{(6)}$ for the stacked sample of type G tape is calculated by assuming that the $S^{\text {sol }}$ value is the same as that for type H6 tape. The estimated $\kappa_{\text {cal }}^{(6)}(T)$ and measured $\kappa_{\text {mes }}^{(1)}$ for type G tape are shown in Fig. $5(\mathrm{~b})$. $\kappa_{\text {cal }}^{(6)}(T)$ is almost equal to $\kappa_{\text {mes }}^{(1)}(T)$, but is larger than $\kappa_{\text {mes }}^{(1)}(T)$ below $20 \mathrm{~K}$, because $\kappa_{\text {mes }}^{\text {sol }}(T)$ is somewhat larger than $\kappa_{\text {mes }}^{(1)}(T)$ at $T<20 \mathrm{~K}$. Weiss et al. [7] reported that the $\kappa(T)$ of a stacked sample consisting of triple Bi2223/Ag$5 \mathrm{wt} \% \mathrm{Au}$ tapes and solder is larger than that of the single tape below $50 \mathrm{~K}$, which shows that our estimation for type G tape is reasonable. Consequently, we conclude that the thermal conductivity of the multi-stacked sample can be estimated using those of the single tape and the solder. 


\section{Summary}

The thermal conductivity, $\kappa(T)$, as a function of temperature for two types of DI-BSCCO tapes was measured. The $\kappa(T)$ for DI-BSCCO sheathed with $\mathrm{Ag}$ of $\mathrm{RRR}=15$ (type $\mathrm{H}$ ) decreases with decreasing temperature, starts to increase below $60 \mathrm{~K}$, and has a large peak around $15 \mathrm{~K}$, at which the peak value is about $800 \mathrm{~W} \mathrm{~m}^{-1} \mathrm{~K}^{-1}$. On the other hand, for DI-BSCCO sheathed with Ag-5.4wt\% Au alloy (type G), $\kappa(T)$ decreases monotonically with decreasing temperature. The magnitude of $\kappa(T)$ for the type $\mathrm{G}$ tape is somewhat smaller than that of the type $\mathrm{H}$ tape.

The $\kappa(T)$ for a stacked sample consisting of six pieces of DI-BSCCO tape sheathed with Ag was measured, which can be compared to the calculated $\kappa(T)$ using the measured $\kappa(T)$ of the single tape and the solder. The measured and calculated $\kappa(T)$ agree well with each other. The $\kappa(T)$ for the stacked sample was also estimated for a DI-BSCCO tape sheathed with Ag-Au5.4wt\% alloy. These results are applicable to other multi-stacked superconducting tapes for cryogenic engineering.

\section{References}

[1] Kobayashi S, Yamazaki K, Kato T, Ohkura K, Ueno E, Fujino K, Fujikami J, Ayai N, Kikuchi M, Hayashi K, Sato K, Hata R. Controlled over-pressure sintering process of Bi2223 tapes. Physica C 2005;426431:1132-1137.

[2] Yamade S, Ayai N, Fujikami J, Kobayashi S, Ueno E, Yamazaki K, Kikuchi M, Kato T, Hayashi K, Sato K, Kitaguchi H, Shimoyama J. De- 
velopment of high performance DI-BSCCO tape with over 200 A critical current. Physica C 2007;463-465:821-824.

[3] Kikuchi M, Kato T, Ohkura K, Ayai N, Fujikami J, Fujino K, Kobayashi S, Ueno E, Yamazaki K, Yamade S, Hayashi K, Sato K, Nagai T, Matsui Y. Recent development of drastically innovative BSCCO wire (DIBISCCO). Physica C 2006;445-448:717-721.

[4] Sasaoka T, Nomura K, Sato J, Kuma S, Fujishiro H, Ikebe M, Noto K. Characteristics of $\mathrm{Ag}-\mathrm{Au}$ alloy sheathed $\mathrm{Bi}-\mathrm{Pb}-\mathrm{Sr}-\mathrm{Ca}-\mathrm{Cu}-\mathrm{O}$ superconducting tapes for current leads. Appl. Phys. Lett. 1994;64:1304-1305.

[5] Fujishiro H, Ikebe M, Noto K, Matsukawa M, Sasaoka T, Nomura K, Sato J, Kuma S. Low thermal conductive Bi-2223 tapes sheathed with Ag-Au alloys. IEEE Trans. Mag. 1994;30:1645-1650.

[6] Putti M, Bellingeri E, Ferdeghini C, Goldacker W, Vase P. Thermal conductivity of silver alloy stabilizers for high temperature superconductor current leads. Supercond. Sci. Technol. 2001;14:L1-3.

[7] Weiss K P, Schwarz M, Lampe A, Heller R, Fietz W H, Nyilas A, Schlachter S I, Goldacker W. Electromechanical and thermal properties of Bi2223 tapes. IEEE Trans. Appl. Supercond. 2007;17:3079-3082.

[8] Nakane H, Watanabe T, Nagata C, Fujiwara S, Yoshizawa S. Measuring the temperature dependence of resistivity of high purity copper using a solenoid coil (SRPM method). IEEE Trans. Instrum. Meas. 1992;41;107110. 
[9] Ikebe M, Fujishiro H, Naito T, Noto K. Simultaneous measurement of thermal diffusivity and conductivity applied to Bi-2223 ceramic superconductors. J. Phys. Soc. Jpn. 1994;63:3107-3114.

[10] Crisp R S, Rungis J. Thermal conductivity in the silver-gold alloy system from 3-300 K. Philos. Mag. 1970;22;217-236. 
Table 1: Specifications of the DI-BSCCO tapes

\begin{tabular}{lccc}
\hline & Type $\mathrm{H}$ & Type $\mathrm{G}$ & Type H6 \\
\hline sheath material & $\mathrm{Ag}(\mathrm{RRR}=15)$ & Ag-5.4wt\%Au & $\mathrm{Ag}(\mathrm{RRR}=15)$ \\
$T_{\mathrm{c}}(\mathrm{K})$ & 110.8 & 111.0 & 111.0 \\
cross section $\left(\mathrm{mm}^{2}\right)$ & 0.935 & 0.994 & 5.88 \\
width $(\mathrm{mm})$ & 4.25 & 4.14 & 4.42 \\
thickness $(\mathrm{mm})$ & 0.22 & 0.24 & $1.33^{*}$ \\
sheath/Bi2223 ratio & 1.8 & 1.8 & - \\
\hline
\end{tabular}

* including the thickness of solder layers. 


\section{Figure captions}

Figure 1

Temperature dependence of the reduced electrical resistance, $R(T) / R(11.5$

$\mathrm{K})$, for $\mathrm{Ag}$ of type $\mathrm{H}$ tape. Inset shows the residual resistance ratio (RRR) vs. impurity content for $\mathrm{Cu}$, which is derived from Ref. [8]. The arrow roughly indicates the impurity content for $\mathrm{RRR}=15$.

Figure 2

Photograph of the cross section of type $\mathrm{H}$ tape. Black and white regions represent the Bi2223 filaments and Ag region, respectively.

Figure 3

Temperature dependence of the thermal conductivity, $\kappa(T)$, for the two types of DI-BSCCO tape (type H, type G). The broken lines represent the electron thermal conductivity, $\kappa_{\mathrm{el}}$, for each sample. The experimental setup is schematically illustrated in the inset, where the coordinate axes represent the crystallographic axes of $\mathrm{Bi} 2223$.

Figure 4

Temperature dependence of the electrical resistivity, $\rho(T)$, for the two types of DI-BSCCO tape (type H, type G).

\section{Figure 5}

(a) Temperature dependence of the thermal conductivities, $\kappa(T)$ 's, for the DI-BSCCO tape sheathed by Ag (H), the sixfold sample (H6), and the solder 
(LFM-48). The calculated $\kappa(T)$ values for the sixfold sample (H6 cal) are also shown. The inset shows a schematic image of the heat flow in the stacked sample, where the coordinate axes represent the crystallographic axes of Bi2223. (b) The estimated $\kappa(T)$ for the sixfold type $\mathrm{G}$ sample (G6), the measured $\kappa(T)$ for the single tape (type G) and the solder (LFM-48) are shown. 


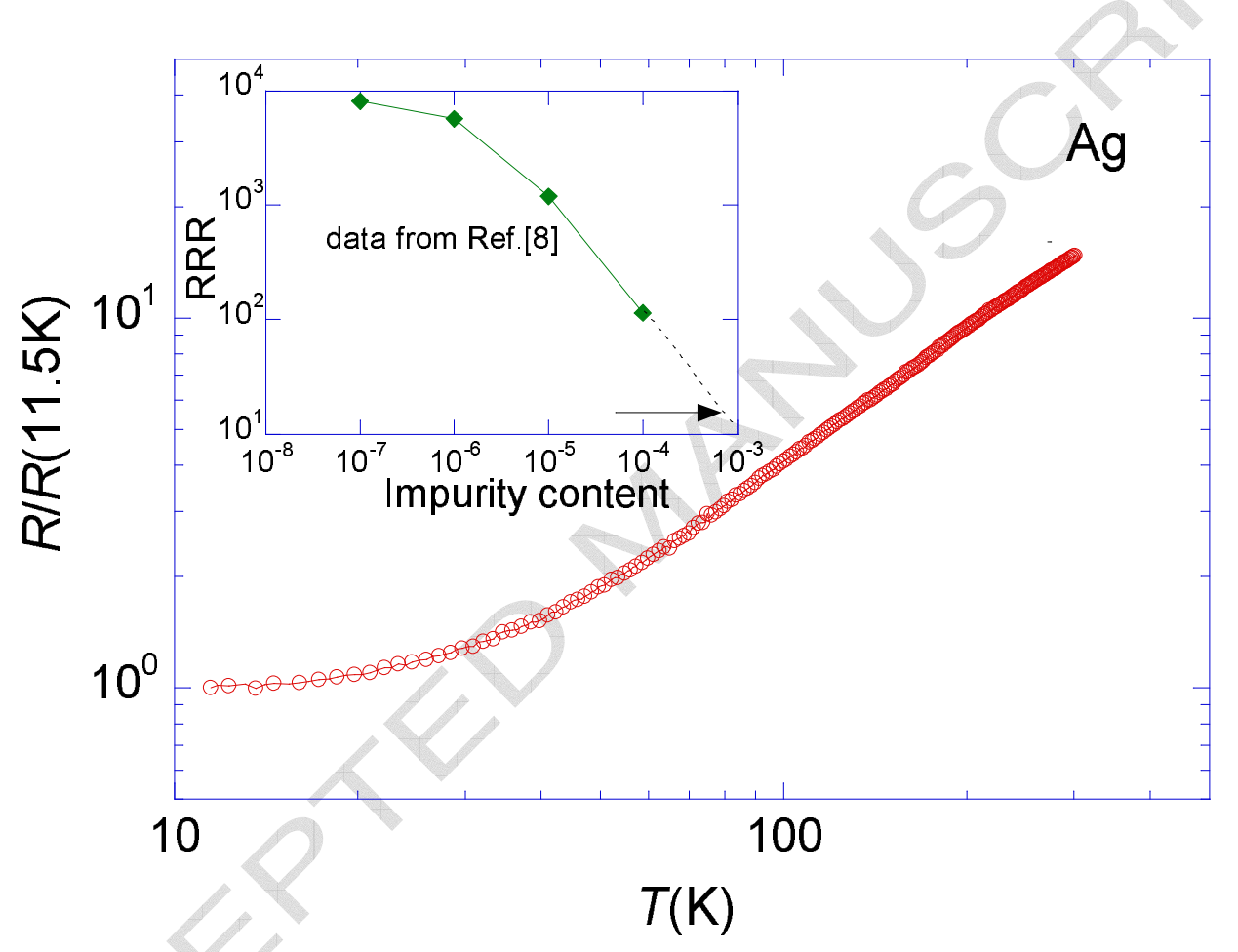

Figure 1: T. Naito et al. 


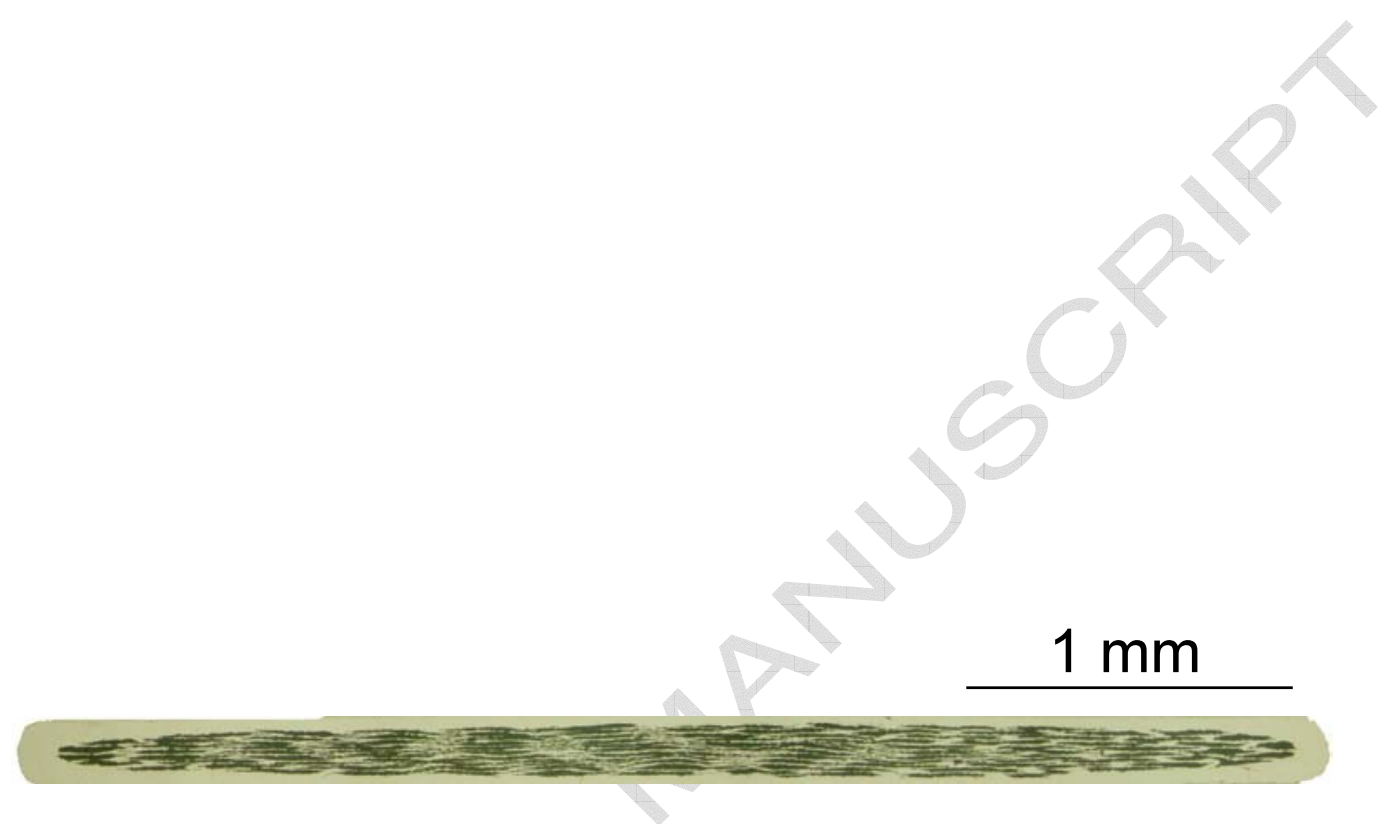

Figure 2: T. Naito et al. 


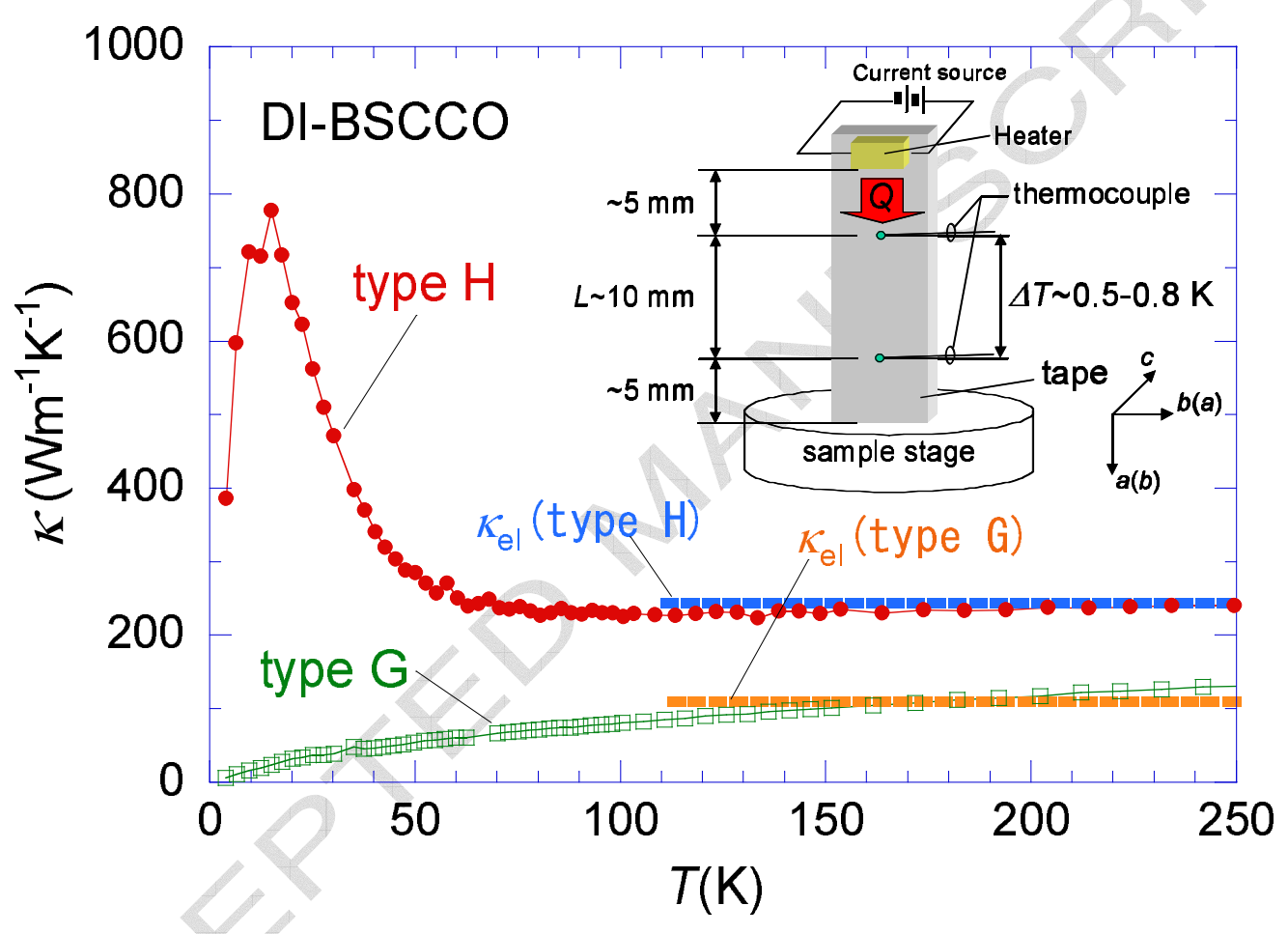

Figure 3: T. Naito et al. 


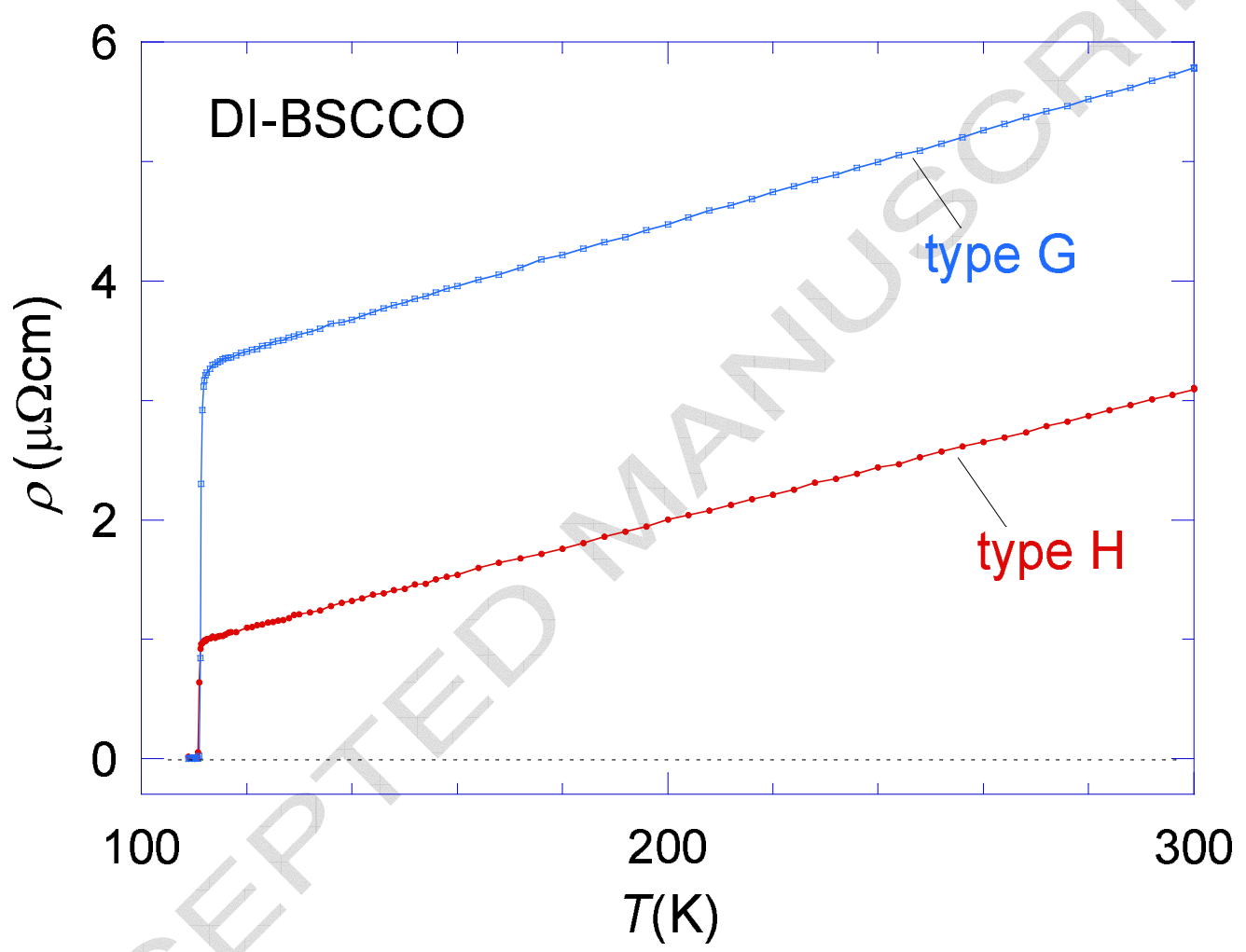

Figure 4: T. Naito et al. 

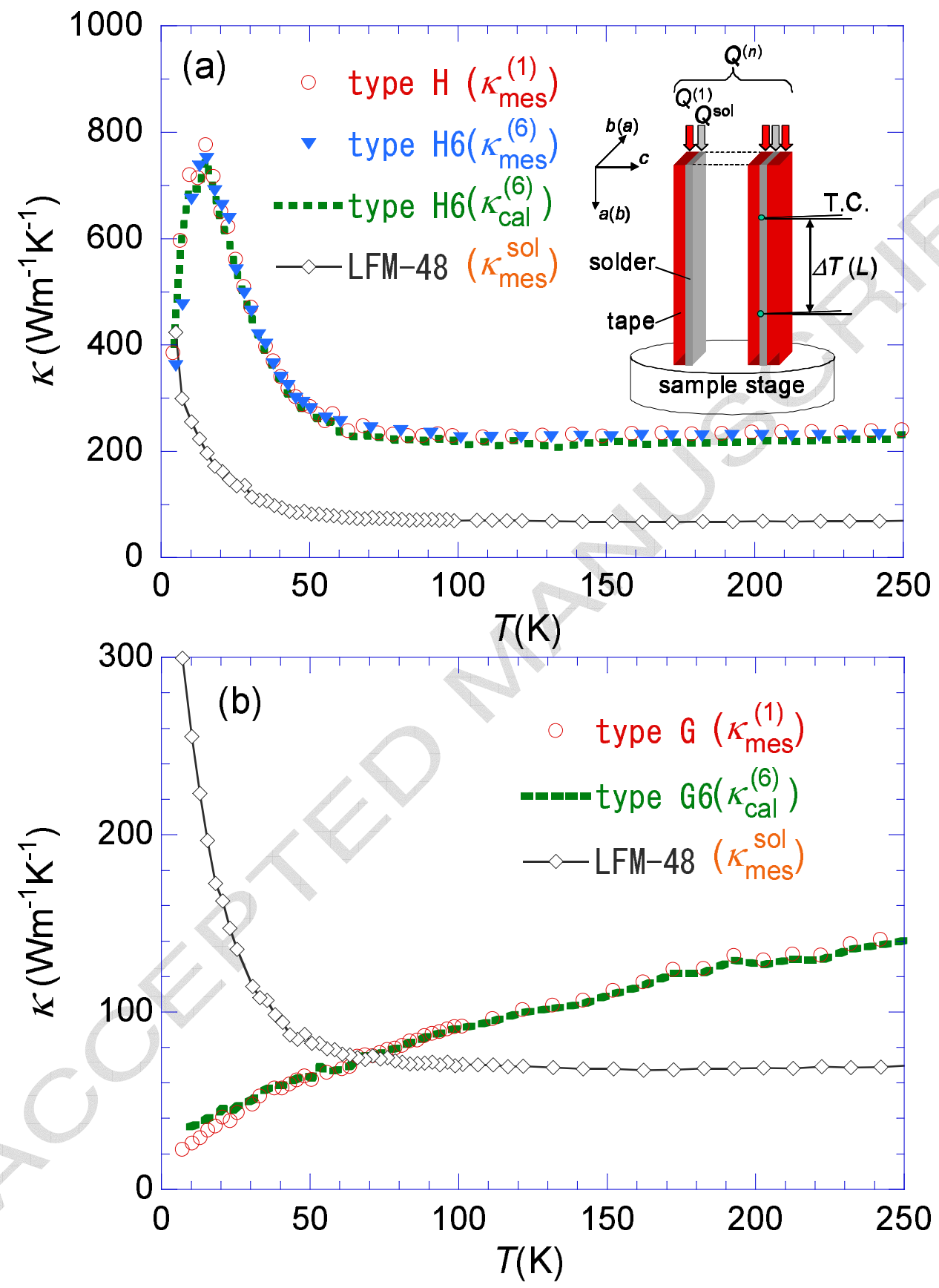

Figure 5: T. Naito et al. 